\title{
INTERACTION BETWEEN LINEAR AND CUBIC PARAMETRIC EXCITATIONS
}

\author{
NGUYEN VAN DINH - TRAN KIM CHI \\ Institute of Mechanics
}

In [1], a quasi-linear oscillating system with non linear restoring element harmonically depending on time has been studied. The mentioned element is represented by two terms-the linear and the cubic. We can consider the system examined as the one subjected to two parametric excitations. The oscillations thus result from the interaction between theșe two excitations.

In the present paper, the same system will be examined in the case general, assuming that there exists certain phase shift between two parametric excitations. As it will be shown, the form of the resonance curve is diversity and can be classified by using critical representative points [2].

\section{$\S 1$ System under consideration and equations of stationary oscillations}

Let us consider a quasilinear system described by the differential equation:

$$
\ddot{x}+\omega^{2} x=\varepsilon\left\{\Delta x-h \dot{x}-\gamma x^{3}+2 p x \cos 2 \omega t+2 q x^{3} \cos 2(\omega t+\sigma)\right\},
$$

where: $x$-oscillatory variable; $h \geq 0$-damping coefficient; $2 p>0,2 q>0$-intensities of linear and cubic parametric excitations, respectively; $2 \omega$-common frequency; $2 \sigma(0 \leq 2 \sigma<2 \pi)$-phase shift; $\varepsilon>0$-small parameter; $\varepsilon \Delta=\left(\omega^{2}-1\right)$-detuning parameter (1-own frequency). mulas

Introducing slowly varying amplitude and dephase $a, \theta$ by means of the for-

$$
x=a \cos \psi ; \quad \dot{x}=-\omega a \sin \psi ; \quad \psi=\omega t+\theta
$$

and applying the averaging method [1], we obtain:

$$
\begin{aligned}
\dot{a} & =\frac{-\varepsilon a}{2 \omega} f=\frac{-\varepsilon a}{2 \omega}\left\{h \omega+p \sin 2 \theta+\frac{1}{2} q a^{2} \sin 2(\theta-\sigma)\right\} \\
a \dot{\theta} & =\frac{-\varepsilon a}{2 \omega} g=\frac{-\varepsilon a}{2 \omega}\left\{\left(\Delta-\frac{3 \gamma}{4} a^{2}\right)+p \cos 2 \theta+q a^{2} \cos 2(\theta-\sigma)\right\} .
\end{aligned}
$$


Stationary oscillations (their constant amplitudes and dephases) satisfy the equations:

$$
\begin{aligned}
& f=h \omega+\left(p+\frac{1}{2} q a^{2} \cos 2 \sigma\right) \sin 2 \theta-\frac{1}{2} q a^{2} \sin 2 \sigma \cos 2 \theta=0 \\
& g=\left(\Delta-\frac{3 \gamma}{4} a^{2}\right)+q a^{2} \sin 2 \sigma \sin 2 \theta+\left(p+q a^{2} \cos 2 \sigma\right) \cos 2 \theta=0 .
\end{aligned}
$$

As in [2], the coefficients of these equations are denoted as:

$$
\begin{aligned}
& A=p+\frac{1}{2} q a^{2} \cos 2 \sigma ; \quad B=-\frac{1}{2} q a^{2} \sin 2 \sigma ; \quad E=-h \omega \\
& G=q a^{2} \sin 2 \sigma ; \quad H=p+q a^{2} \cos 2 \sigma ; \quad K=\frac{3 \gamma}{4} a^{2}-\Delta .
\end{aligned}
$$

\section{§2. Critical region, compatible ensemble and trigonometri- cal conditions}

Depending on $\sigma$, various forms of the resonance curve will be obtained. Before analyzing, it is necessary to determine the critical region and the critical part of the resonance curve. Three characteristic determinants are:

$$
\begin{aligned}
D & =\left|\begin{array}{ll}
A & B \\
G & H
\end{array}\right|=\left(p+\frac{1}{2} q a^{2} \cos 2 \sigma\right)\left(p+q a^{2} \cos 2 \sigma\right)+\frac{1}{2} q^{2} a^{4} \sin ^{2} 2 \sigma \\
& =p^{2}+\frac{3}{2} q a^{2} \cos 2 \sigma+\frac{1}{2} q^{2} a^{4}, \\
D_{1} & =\left|\begin{array}{ll}
E & B \\
K & H
\end{array}\right|=\frac{1}{2} q a^{2}\left(\frac{3 \gamma}{4} a^{2}-\Delta\right) \cdot \sin 2 \sigma-h \omega q a^{2} \sin 2 \sigma \\
D_{2} & =\left|\begin{array}{ll}
A & E \\
G & K
\end{array}\right|=\left(p+\frac{1}{2} q a^{2} \cos 2 \sigma\right)\left(\Delta-\frac{3 \gamma}{4} a^{2}\right)+h \omega q a^{2} \sin 2 \sigma .
\end{aligned}
$$

The critical region $R_{2}\left(\Delta, a^{2}\right)$ satisfy the equality:

$$
D=0 \text { i.e. } \frac{1}{2} q^{2} a^{4}+\frac{3}{2} p q a^{2} \cos 2 \sigma+p^{2}=0 .
$$

Being a quadratic equation with unknown $a^{2},(2.2)$ admits two positive roots if:

$$
9 \cos ^{2} 2 \sigma-8 \geq 0, \quad \cos 2 \sigma<0
$$

i.e:

$$
-1 \leq \cos 2 \sigma \leq-\frac{2 \sqrt{2}}{3} \text { or } \frac{\pi}{2}-\bar{\sigma} \leq \sigma \leq \frac{\pi}{2}+\bar{\sigma}
$$


where

$$
\bar{\sigma}=\frac{1}{2} \arccos \frac{2 \sqrt{2}}{2},
$$

$\sigma$ satisfying-(2.4), the critical-region exists and consists of two straight linesdenoted by $D^{\prime}, D^{\prime \prime}$ parallel to the abscissa axis $\Delta$ with ordinates:

$$
\begin{aligned}
& D^{\prime}: a_{1}^{2}=-\frac{3 p}{2 q}\left(-\cos 2 \sigma-\sqrt{\cos ^{2} 2 \sigma-\frac{8}{9}}\right), \\
& D^{\prime \prime}: a_{2}^{2}=\frac{3 p}{2 q}\left(-\cos 2 \sigma+\sqrt{\cos ^{2} 2 \sigma-\frac{8}{9}}\right)
\end{aligned}
$$

(a "double" line if $\cos ^{2} 2 \sigma=\frac{8}{9}$ i.e. $\sigma=\frac{\pi}{2} \pm \bar{\sigma}$ ) In these two critical lines, the compatible ensemble is determined by two compatibility conditions:

$$
\begin{aligned}
& D_{1}=0 \text { i.e. } \frac{1}{2} q a^{2}\left(\frac{3 \gamma}{4} a^{2}-\Delta\right) \sin 2 \sigma-h \omega\left(p+q a^{2} \cos 2 \sigma\right)=0 \\
& D_{2}=0 \text { i.e. } h \omega q a^{2} \sin 2 \sigma+\left(\frac{3 \gamma}{4} a^{2}-\Delta\right)\left(p+\frac{1}{2} q a^{2} \cos 2 \sigma\right)=0 .
\end{aligned}
$$

Then, from the compatible ensemble obtained, we single out the critical part $C_{2}$ (of the resonance curve $C$ ) by two trigonometrical conditions:

$$
\begin{gathered}
A^{2}+B^{2} \geq E^{2} \text { i.e. }\left(p+\frac{1}{2} q a^{2} \cos 2 \sigma\right)^{2}+\frac{1}{4} q^{2} a^{2} \sin ^{2} 2 \sigma \geq h^{2} \omega^{2}, \\
G^{2}+H^{2} \geq K^{2} \text { i.e. } q^{2} a^{4} \sin ^{2} 2 \sigma+\left(p+q a^{2} \cos 2 \sigma\right)^{2} \geq\left(\frac{3 \gamma}{4} a^{2}-\Delta\right)^{2} .
\end{gathered}
$$

The resonance curve $C$ will be identified from the following frequency-amplitude relationship:

$$
\begin{aligned}
W\left(\Delta, a^{2}\right)= & D_{1}^{2}+D_{2}^{2}-D^{2}= \\
= & \left\{\frac{1}{2} q a^{2}\left(\frac{3 \gamma}{4} a^{2}-\delta\right) \sin 2 \sigma+h \omega\left(p+q a^{2} \cos 2 \sigma\right)\right\}^{2}+ \\
& +\left\{h \omega q a^{2} \sin 2 \sigma+\left(\frac{3 \gamma}{4} a^{2}-\Delta\right)\left(p+\frac{1}{2} q a^{2} \cos 2 \sigma\right)\right\}^{2} \\
& -\left\{\left(p+\frac{1}{2} q a^{2} \cos 2 \sigma\right)\left(p+q a^{2} \cos 2 \sigma\right)-\frac{1}{2} q^{2} a^{4} \sin ^{2} 2 \sigma\right\}^{2}=0
\end{aligned}
$$

with condition that compatible points do not satisfying trigonometrical conditions must be rejected. 


\section{§3. The case $\sigma=\pi / 2$}

This particular case has been studied in [1]. Here, it will be examined again, insisting on the critical part $C_{2}$.

As we have already known, the resonance curve $C$ consists of two parts:

- the ordinary part $C_{1}$, located in the ordinary region $D \neq 0$ and

- the critical part $C_{2}$, located in the critical region $D=0$.

In the case $\sigma=\pi / 2$, the ordinates of two critical lines are:

$$
D^{\prime}: a_{1}^{2}=p / q ; \quad D^{\prime \prime}: a_{2}^{2}=\frac{2 p}{q}
$$

and two compatibility conditions $(2.7)(2.8)$ become simple:

$$
\begin{array}{ll}
D_{1}=0 & \text { i.e. } \quad h \omega\left(p-q a^{2}\right)=0 \\
D_{2}=0 & \text { i.e. } \quad\left(\frac{3 \gamma}{4} a^{2}-\Delta\right)\left(p-\frac{1}{2} q a^{2}\right)=0
\end{array}
$$

If $h=0$ (system without damping), (3.2) is "automatically" satisfied, (3.3) is also satisfied on $D^{\prime \prime}$ but on $D^{\prime},(3.3)$ is only satisfied if $\Delta=\frac{3 \gamma}{4} a_{1}^{2}$. Thus, for the system without damping, the compatible ensemble consists of the critical line $D^{\prime \prime}$ and the point $I\left(\Delta=\frac{3 \gamma}{4} a_{1}^{2} ; a^{2}=a_{1}^{2}\right)$ in $D^{\prime}$.

Examining two trigonometrical conditions, it is easy to verify that:

- At I, these two conditions are satisfied since all two right hand sides of (2.9) (2.10) vanish;

- On $D^{\prime \prime},(2.9)$ is always satisfied but (2.10) is only satisfied if

$$
\left(\Delta-\frac{3 \gamma}{4} a_{2}^{2}\right)^{2}<p^{2} \text { i.e. } \frac{3 \gamma}{4} a_{2}^{2}-p \leq \Delta \leq \frac{3 \gamma}{4} a_{2}^{2}+p .
$$

Thus, the critical part $C_{2}$ (in the case $h=0$ ) consists of the critical point I and the critical segment $J_{1} J_{2}$ in $D^{\prime \prime}$

$$
\frac{3 \gamma}{4} a_{2}^{2}-p \leq \Delta \leq \frac{23 \gamma}{4} a_{2}^{2}+p ; \quad a^{2}=a_{2}^{2}
$$

If $h>0$ (system with damping), the two compatibility conditions are only satisfied at I i.e the compatible ensemble is reduced to $I$. At this unique compatible point $I,(2.10)$ is always satisfied, but (2.9) requires:

$$
h^{2} \leq \frac{p^{2}}{4 \omega_{1}^{2}}, \quad \omega_{1}^{2}=\frac{3 \gamma}{4} a_{1}^{2}+1
$$


So, $I$ is only critical if the damping is weak enough.

In Fig. 1, the resonance curves have been plotted for fixed values $\sigma=\pi / 2$; $p=0.01, q=0.02, \gamma=0.03$ and for various values of $h$. The point $\mathrm{I}$, the segment $J_{1} J_{2}$ and two straight-lines-(1) form-the resonance curve of the system without damping $(h=0)$. The resonance curves $(2),(3)$ correspond to $h=0.001 ; h=0.005$ respectively. We see that, for $h>0$ (system with damping), the critical segment. $J_{1} J_{2}$ disappears and the resonance curve separates itself into two branches-the upper and the lower. The upper branch of "hyperbolic" form, moves up as $h$ increases. The form of the lower branch depends on $h$. If $h$ is small enough, the lower branch intersects the abscissa axis. at two points, contains the nodal point I and has a loop. Increasing $h$, the loop becomes narrower then disappears, the nodal point I degenerates into a returning point then disappears (an isolated point), the lower branche takes the form of a "parabola top". This "top" moves down as $h$ increases, then finally disappears when $h$ is large enough.

Fig. 1

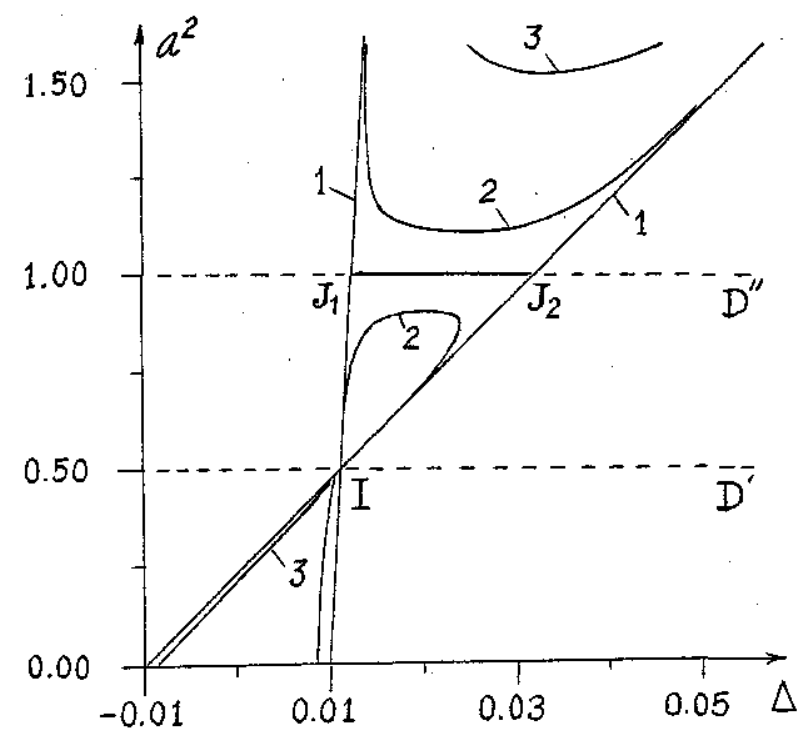

§4. The case $\frac{\pi}{2} \neq \sigma \in\left[\frac{\pi}{2}-\bar{\sigma}, \frac{\pi}{2}+\bar{\sigma}\right]$

This case is characterized by two critical points. If $h=0$ (system without damping), the two compatibility conditions become:

$$
\begin{array}{ll}
D_{1}=0 & \text { i.e. } \quad \frac{1}{2} q a^{2}\left(\frac{3 \gamma}{4} a^{2}-\Delta\right) \sin 2 \sigma=0 \\
D_{2}=0 & \text { i.e. } \quad\left(\frac{3 \gamma}{4} a^{2}-\Delta\right)\left(p+\frac{1}{2} q a^{2} \cos 2 \sigma\right)=0
\end{array}
$$


It is easy to prove that there exists two compatible points:

$$
\begin{array}{lll}
\text { in } D^{\prime}: & I_{0}: \Delta=\Delta_{10}=\frac{3 \gamma}{4} a_{1}^{2} ; & a^{2}=a_{1}^{2}, \\
\text { in } D^{\prime \prime}: & J_{0}: \Delta=\Delta_{20}=\frac{3 \gamma}{4} a_{2}^{2} ; & a^{2}=a_{2}^{2} .
\end{array}
$$

All two trigonometrical conditions (2.9), (2.10) are evidently satisfied; hence, both $I_{0}, J_{0}$ are critical

If $h>0$ (system with damping), in each critical line, there exists a unique compatible point, too

Indeed, let us examine-for instance-the critical line $D^{\prime}$. Substituting $\Delta$ by $\omega^{2}-1$, the first compatibility condition (2.7) becomes a quadratic equation with unknown $\omega$ :

$$
\omega^{2} \cdot q a_{1} \sin 2 \sigma+2 h \omega\left(p+q a_{1}^{2} \cos 2 \sigma\right)-q a_{1}^{2}\left(\frac{3 \gamma}{4} a_{1}^{2}+1\right) \sin 2 \sigma=0
$$

This equation admits a positive root:

$$
\omega_{1}=\frac{1}{q a_{1}^{2} \sin 2 \sigma}\left\{-h\left(p+q a_{1}^{2} \cos 2 \sigma\right) \pm \sqrt{Z}\right\}
$$

where:

$$
Z=h^{2}\left(p+q a_{1}^{2} \cos 2 \sigma\right)^{2}+q^{2} a_{1}^{4} \sin ^{2} 2 \sigma\left(\frac{3 \gamma}{4} a_{1}^{2}+1\right) .
$$

The sign $+(-)$ corresponds to $2 \sigma>0(<0)$.

The compatible point in $D^{\prime}$-denoted by I-has as coordinates:

$$
I: \Delta=\Delta_{1}=\omega_{1}^{2}-1 ; \quad a^{2}=a_{1}^{2} .
$$

If $h=0, \omega_{1}=\sqrt{\frac{3 \gamma}{4} a_{1}^{2}+1}, \Delta_{1}=\omega_{1}^{2}-1=\frac{3 \gamma}{4} a_{1}^{2}=\Delta_{10}$ i.e. I coincides with $I_{0}$. Increasing $h$, the compatible point I moves along $D^{\prime}$ (since $a_{1}^{2}$ remains constant), to the right (left) if $\sin 2 \sigma>0(<0)$. Indeed, we have:

$$
\frac{d \omega_{1}}{d h}=\frac{\left(p+q a_{1}^{2} \cos 2 \sigma\left\{ \pm h\left(p+q a_{1}^{2} \cos 2 \sigma\right)-\sqrt{Z}\right\}\right.}{q a_{1}^{2} \sin 2 \sigma \cdot \sqrt{Z}}
$$

In the case $\sin 2 \sigma>0$ ( $\operatorname{sign}+$ before $h$ ), the denominator of (4.9) is positive, the sum in the curly bracket of the numerator is negative; therefore, the sign of $\frac{d \omega_{1}}{d h}$ is the same as that of (in view of the expression of $a_{1}^{2}$ ):

$$
-\left(p^{2}+q a_{1}^{2} \cos 2 \sigma\right)=\frac{p}{2}\left\{\left(3 \cos ^{2} 2 \sigma-2\right)+3 \cos 2 \sigma \sqrt{\cos ^{2} 2 \sigma-\frac{8}{9}} .\right.
$$


Since $-1<\cos 2 \sigma \leq \frac{-2 \sqrt{2}}{3}, 3 \cos ^{2} 2 \sigma-2>0,-3 \cos 2 \sigma>0$, the inequatily:

$\left(3 \cos ^{2} 2 \sigma-2\right)+3 \cos 2 \sigma \sqrt{\cos ^{2} \sigma-\frac{8}{9}}>0$ i.e. $3 \cos ^{2} 2 \sigma-2>-3 \cos 2 \sigma \sqrt{\cos ^{2} 2 \sigma-\frac{8}{9}}$

l̂eads to

$$
\left(3 \cos ^{2} 2 \sigma-2\right)^{2}>9 \cos ^{2} 2 \sigma\left(\cos ^{2} \sigma-\frac{8}{9}\right) \text { i.e. } 4>4 \cos ^{2} 2 \sigma .
$$

So, $\frac{d \omega_{1}}{d h}>0$, I moves to the right.

In the case $\sin 2 \sigma<0$, I moves to the left.

Let us verify two trigonometrical conditions (2.9) (2.10). The second condition can be written as:

$$
\Delta_{1}^{-}=\frac{3 \gamma}{4} a_{1}^{2}-\sqrt{Y} \leq \Delta \leq \frac{3 \gamma}{4} a_{1}^{2}+\sqrt{Y}=\Delta_{1}^{+}
$$

where:

$$
Y=q^{2} a_{1}^{4} \sin ^{2} 2 \sigma+\left(p+q a_{1}^{2} \cos 2 \sigma\right)^{2}
$$

This "double" inequality shows that, as $h$ increases the critical point $I$, starting from $I_{0}$, moves along $D^{\prime}$ (to the right if $\sin 2 \sigma>0$ and to the left if $\sin 2 \sigma<0$ ) will be degenerated into a "banal" compatible point when $h$ exceeds certain values (corresponding to $\Delta_{1}^{+}$or $\Delta_{1}^{-}$). The first trigonometrical condition gives:

$$
h^{2} \leq \frac{\left(p+\frac{1}{2} q a_{1}^{2} \cos 2 \sigma\right)^{2}+\frac{1}{4} q^{2} a_{1}^{4} \sin ^{2} 2 \sigma}{\frac{3 \gamma}{4} a_{1}^{2}+1 \pm \sqrt{Y}} .
$$

The same method can be applied to study the compatible point $J$ in $D^{\prime \prime}$.

In Fig. 2, for fixed values $\sigma=1.48>\bar{\sigma} ; p=0.01, q=0.02 ; \dot{\gamma}=0.01$, the resonance curves (1) (2) (3) correspond to $h=0, h=0.002, h=0.005$ respectively. The curve (1) has two critical nodal points $I \equiv I_{0}, J \equiv J_{0}$. As $h$ increases, these two points move to the right. The nodal point $J$ first disappears and the resonance curve is divided into two branches-the upper and the lower.

\section{§5. The case $\sigma \notin\left[\frac{\pi}{2}-\bar{\sigma}, \frac{\pi}{2}+\bar{\sigma}\right]$}

In this case, the critical region and, consequently, the critical part $C_{2}$ of the resonance curve does not exist; we have only the ordinary part $C_{1}$. 


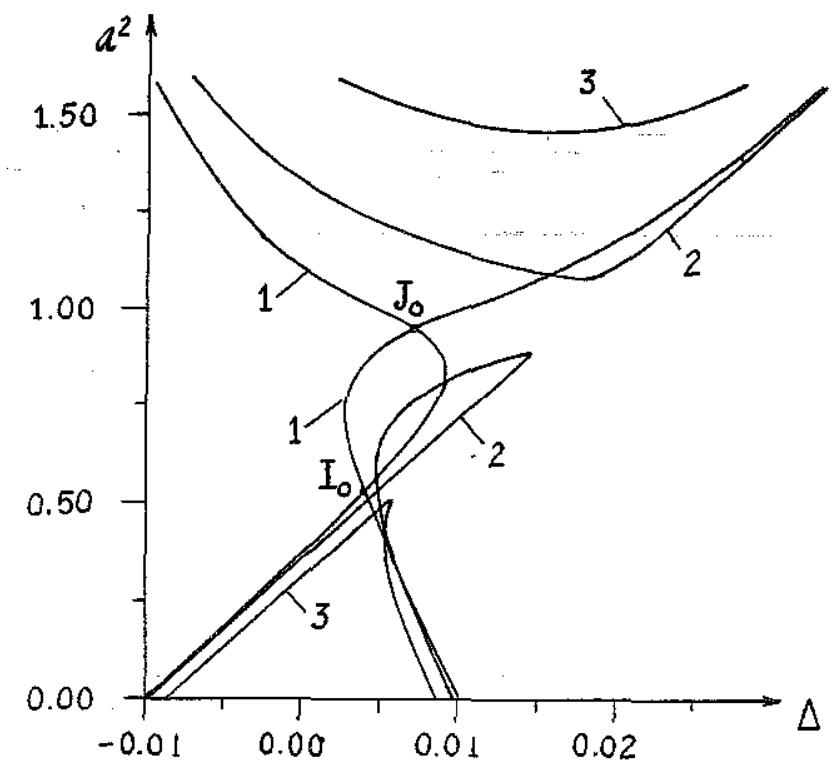

Fig. 2

If $h=0$, the resonance curve consists of two branches (all these two branches belong to $C_{1}$ ) respectively located in the left and right hand sides of the separating line $\Delta=\frac{3 \gamma}{4} a^{2}$. As $h$ increases, these two branches approach each other. They will connect with each other at a certain singular point (this point belongs to $C_{1}$ too) when $h$ reaches certain value $h_{*}$, as $h$ exceeds $h_{*}$ the resonance curve is divided into two branches-the lower and the upper.

In Fig. 3, the resonance curve has been plotted for fixed values $\sigma=1.4<\bar{\sigma}$; $p=0.01 ; q=0.02 ; \gamma=0.01:$ (1) (2) (3) correspond to $h=0 ; h=0.00511 ; 0.0055$ respectively; the aforesaid singular point appears when $h \approx 0.00511$.

\section{$\S 6$. Stability conditions}

The stability study is based on the variational system and its characteristic equation. Two stability conditions are of well-known form:

$$
S_{1}=a \frac{\partial f}{\partial a}+\frac{\partial g}{\partial \theta}>0 ; \quad S_{2}=\frac{\partial f}{\partial a} \frac{\partial g}{\partial \theta}-\frac{\partial f}{\partial \theta} \frac{\partial a}{\partial a}>0 .
$$

The first stability condition can be transformed as:

$$
\begin{aligned}
S_{1} & =2\left\{a^{2} \frac{\partial f}{\partial a^{2}}+\frac{\partial g}{\partial(2 \theta)}\right\}=2\left\{a^{2} \cdot \frac{1}{2} q \sin 2(\theta-\sigma)-p \sin 2 \theta-q a^{2} \sin 2(\theta-\sigma)\right\} \\
& =2\left\{-p \sin 2 \theta-\frac{1}{2} q a^{2} \sin 2(\theta-\sigma)\right\}=2 h \omega>0 .
\end{aligned}
$$




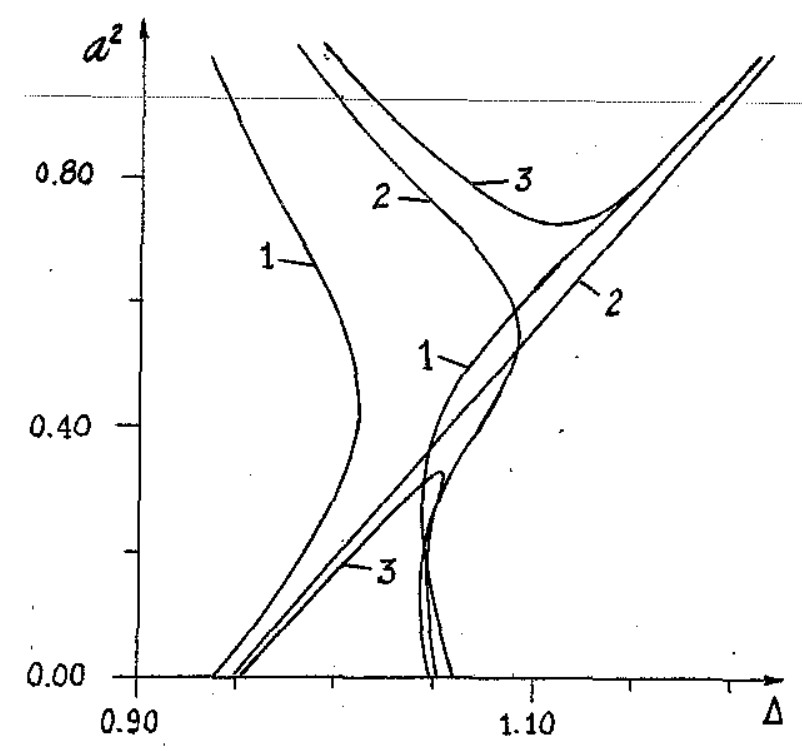

Fig. 9

Thus, only the system with damping satisfies the first stability condition. $[3]:$

For the second stability condition, it is convenient to use its compact form

$$
S_{2}=\frac{1}{2 D} \frac{\partial W}{\partial a}=\frac{a}{D} \frac{\partial W}{\partial a^{2}}>0
$$

The geometrical interpretation of (6.3) is as follows: in the plane $R\left(\Delta, a^{2}\right)$, moving up parallel to the ordinate axis $a^{2}$, if from a domain $D \cdot W<0(>0)$, crossing the ordinary part $C_{1}$, we enter into that $D \cdot W>0(<0)$, the crossing point corresponds to stable (unstable) stationary oscillation.

For a critical stationary oscillation represented by the critical point $I$ considered as an element of the ordinary branch (i), its stability is the same as that of the branch (i)

\section{Conclusion}

The analyses presented show the role of the phase shift between two parametric excitations. The existence and the structure of the critical part $C_{2}$ depend on the phase shift. Consequently, various forms of the resonance curve are obtained.

This publication is completed with financial support from The council for Natural Sciences of Vietnam 


\section{References}

1. Mitropolski Yu. A., Nguyen Van Dao. Applied asymptotic method in non linear oscillations. Kluwer Academic Publishers, 1997.

2. Nguyen Van Dinh. Stationary oscillations in degenerated cases. Journal of Mechanics, NCNST of Vietnam, T.XVIII, No. 2, 1996, 13-19.

3. Nguyen Van Dinh. Stability of critical stationary oscillations. Journal of Mechanics, NCNST of Vietnam, T.XIX, No. 2, 1997, 15-20.

Received September 15, 1998

TƯƠNG TÁC GIŨA HAI KÍCH ĐộNG THÔNG SỐ BẬC NHẤT VÀ BẬC BA

Xét hệ á tưyến chịu hai kích động thông số bậc nhất và bậc ba khi có lệch pha giữa hai kích động. Đã phân tích ảnh hưởng của sự lệch pha đó đến sự tồn tại và cấu trúc của phần tới hạn của đường cộng hường và qua đó đến các dạng đường cộng hướng. 